\title{
Repetitive transcranial magnetic stimulation is as effective as fluoxetine in the treatment of depression in patients with Parkinson's disease
}

\author{
F Fregni, C M Santos, M L Myczkowski, R Rigolino, J Gallucci-Neto, E R Barbosa, K D Valente, \\ A Pascual-Leone, M A Marcolin
}

\begin{abstract}
Objective: To study the efficacy of $15 \mathrm{~Hz}$ repetitive transcranial magnetic stimulation (rTMS) in treating depression in patients with Parkinson's disease.

Methods: 42 patients were enrolled into two groups: group 1, active rTMS (15 Hz rTMS for 10 days) and placebo drug treatment; group 2, sham rTMS and fluoxetine $20 \mathrm{mg} /$ day. A specially designed sham coil was used for sham stimulation. The unified Parkinson's disease rating scale (UPDRS), activities of daily living (ADL), Hamilton rating scale for depression (HRSD), Beck depression inventory (BDI), and mini-mental state examination (MMSE) were assessed by a rater blinded to treatment arm.

Results: HRSD and BDI were improved to the same extent in both groups after two weeks of treatment (38\% and $32 \%$ for group 1, 41\% and 33\% for group 2, respectively). At week 8 there was a tendency for worse motor UPDRS scores in group 2 (NS). ADL showed improvement at week 8 only in group 1. MMSE improved in both groups after treatment, but faster in group 1 than in group 2. There were fewer adverse effects in group 1 than in group 2.

Conclusions: rTMS has the same antidepressant efficacy as fluoxetine and may have the additional advantage of some motor improvement and earlier cognitive improvement, with fewer adverse effects.
\end{abstract}

$\mathrm{T}$ ranscranial magnetic stimulation (rTMS) is a noninvasive, well tolerated technique for stimulating the brain. ${ }^{1}$ Many studies have reported antidepressive properties of rTMS applied for two weeks in patients with major depression, ${ }^{2-4}$ though some failed to find a difference with sham stimulation. ${ }^{5}{ }^{6}$ rTMS may have an antidepressant effect by the enhancing the serotoninergic system and increasing monoamine levels. ${ }^{78}$ Furthermore, previous studies showed that rTMS applied to the prefrontal area led to an improvement in motor function in patients with Parkinson's disease. ${ }^{9-11}$ The only study to date that investigated the use of rTMS to treat depression in Parkinson's disease was an open trial that showed a significant decrease in Hamilton scores after 10 days of slow rTMS. ${ }^{11}$ We report here the results of a double blind, sham stimulation controlled, randomised study on 42 patients with Parkinson's disease and depression.

\section{METHODS}

\section{Study population}

We studied 42 patients with idiopathic Parkinson's disease and major or minor depression according to the UK Parkinson's Disease Brain Bank and the Diagnostic and
Statistical Manual of Mental Disorders, 4th edition (DSM-IV) criteria, respectively (in making the diagnosis of depression in Parkinson's disease, psychomotor retardation was not considered, as previously suggested ${ }^{12}$ ). Patients were excluded if they had been using antidepressants within two months of the study or had ferromagnetic metallic implants, a history of seizures, major head trauma, dementia, or depression with psychotic symptoms. The study was approved by the local ethics committee.

The patients were randomly assigned according to a computer generated randomisation list to one of two groups: group 1: active rTMS and placebo drug treatment (21 patients); group 2: sham rTMS and fluoxetine $20 \mathrm{mg} /$ day (21 patients).

\section{Psychiatric and neurological evaluation}

The psychiatric and neurological raters, who remained blind to the results of the other raters and to the study group assignment, carried out the evaluations before and after two and eight weeks of treatment. Psychiatric evaluation included the Hamilton rating scale for depression (HRSD), the Beck depression inventory (BDI), and the mini-mental state examination (MMSE). Neurological evaluation included the Hoehn and Yahr (HY) scale, the unified Parkinson's disease rating scale (UPDRS, part III), and the Schwab and England activities of daily living index (ADL).

\section{Adverse effects evaluation}

Comprehensive Udvalg for Kliniske Undersogelser (UKU) side effect rating scales ${ }^{13}$ were completed for all patients after two and eight weeks of treatment.

\section{Magnetic stimulation}

Focal rTMS of the left dorsolateral prefrontal cortex was undertaken using a figure-of-eight coil and a Dantec stimulator. Forty trains of five seconds each, using an intensity of $110 \%$ of motor threshold and $15 \mathrm{~Hz}$ frequency, were applied in each patient for 10 days during a two week period. Given that the effects of higher frequencies can last longer than those of lower frequencies, ${ }^{14}$ and $20 \mathrm{~Hz}$ would be higher than the safety guidelines recommended for a five seconds train, ${ }^{15}$ we decided to use a frequency of $15 \mathrm{~Hz}$. For the sham treatment group, stimulation parameters were the same but a sham coil was used.

Abbreviations: ADL, activities of daily living; $B D I$, Beck depression inventory; HRSD, Hamilton rating scale for depression; HY, Hoehn and Yahr scale; MMSE, mini-mental state examination; rTMS, repetitive transcranial magnetic stimulation; SSRI, selective serotonin reuptake inhibitor; UPDRS, unified Parkinson's disease rating scale 


\section{Drug treatment}

Patients of both groups were instructed to take one pill a day. Pills were fluoxetine $20 \mathrm{mg}$ and glucose (placebo) for group 2 and 1 , respectively. We preferred a selective serotonin reuptake inhibitor (SSRI) antidepressant class rather than tricyclic antidepressants, as the latter can aggravate Parkinson's disease associated orthostatic hypotension and exacerbate the cognitive problems. ${ }^{16}$ Several studies have suggested that SSRIs are effective for the treatment of depression in Parkinson's disease, although most have been open studies. ${ }^{16}$

\section{Data analysis}

Baseline comparisons carried out between the two treatment groups were analysed using $\chi^{2}$, Fisher's exact, and Student's $t$ tests. Changes in the scores with treatment were tested using repeated measures analysis of variance (ANOVA). When there was a significant overall effect, post hoc analyses were made between groups using the Tukey-HSD method.

\section{RESULTS}

Selected demographic, psychiatric, and parkinsonian characteristics are shown in table 1. No significant differences were seen between the study groups. All patients completed the study; only one patient who had started the study was excluded owing to an unrelated intestinal infection. All the patients came to 10 rTMS sessions and the pills were taken on a regular basis as shown by the empty pill containers returned at the end of the study.

To assess blinding integrity, interviewers were asked to guess the treatment group of each patient and score their confidence on a scale 1 to 5 (1, totally uncertain; 5 , almost sure). The psychiatric and neurology evaluator guessed correctly in $56 \%$ and $71 \%$ of cases, with a low confidence (mean (SD), $2.6(1.2)$ and 2.9 (1.0), respectively) and low inter-rater agreement $(\kappa=0.02)$.

Two-factor repeated measures ANOVA (subject group, time of evaluation) showed a significant effect of time for HDRS and BDI scores $(\mathrm{F}=36.43, \mathrm{p}<0.001$ and $\mathrm{F}=20.43, \mathrm{p}<0.001$, respectively). Post hoc analysis showed a significant difference between T0 and T2, but not between $\mathrm{T} 2$ and T8, suggesting a long lasting antidepressive effect in both groups. The mean decrease in HDRS and BDI after two weeks of treatment was 38\% (9.5 (8.0)) and 32\% (8.1 (9.2)) for group 1 , and $41 \%(10.5(6.9))$ and $33 \%(8.1(8.3))$ for group 2 , respectively. In each group, nine patients $(43 \%)$ could be classified as treatment responders $(50 \%$ reduction in HDRS scores) after two weeks of treatment. Taking account of the patient excluded, intention to treat analysis showed nine of $22(41 \%)$ and nine of $21(43 \%)$ treatment responders in groups 1 and 2, respectively.

Although group 2 had an unequal sex distribution, repeated measures for ANOVA (sex group, time of evaluation) showed no significant difference between the sexes in the antidepressant response $(\mathrm{F}=0.49, \mathrm{p}=0.61$ for group 2, and $\mathrm{F}=0.55, \mathrm{p}=0.58$ for group 1$)$.

Repeated measures ANOVA for UPDRS scores showed no group $(\mathrm{F}=1.186, \mathrm{p}=0.283)$, time $(\mathrm{F}=1.303, \mathrm{p}=0.278)$, or group $\times$ time effect $(F=0.341, p=0.712)$. Although the effect did not achieve significance, group 1 tended to be stable during the treatment (UPDRS, $\mathrm{T} 0=34.6$ (15.3); $\mathrm{T} 2=34.0$ (16.9); T8 $=34.5(15.6)$ ), while the scores in group 2 tended to worsen after eight weeks (UPDRS, T0 $=38.1$ (17.8), $\mathrm{T} 2=37.8(18.4), \mathrm{T} 8=40.1(17.6))($ fig $1 \mathrm{~A})$.

Table 1 Descriptive characteristics of patient groups

\begin{tabular}{|c|c|c|c|}
\hline & Group 1 ( $n=21)$ & Group $2(n=21)$ & p Value \\
\hline Age (years) & $65.3(7.8)$ & $66.0(8.5)$ & $0.779^{*}$ \\
\hline Male (n) & 11 & 15 & $0.204 \dagger$ \\
\hline Female (n) & 10 & 6 & \\
\hline MMSE & $24.8(4.5)$ & $24.1(3.6)$ & $0.597^{*}$ \\
\hline \multicolumn{4}{|l|}{ Depression features } \\
\hline HDRS & $25.6(6.5)$ & $25.6(7.5)$ & $0.775^{*}$ \\
\hline BDI & $25.1(9.7)$ & $24.5(8.8)$ & $0.843^{*}$ \\
\hline Major depression (n) & 17 & 16 & $1.000 \ddagger$ \\
\hline Minor depression (n) & 4 & 5 & \\
\hline \multicolumn{4}{|l|}{ Motor threshold, mean } \\
\hline Initial (\% output TMS) & $57.0(11.1)$ & $54.4(8.5)$ & $0.774^{*}$ \\
\hline \multicolumn{4}{|l|}{ Parkinsonian features } \\
\hline Age at onset (years) & $58.8(8.5)$ & $57.5(8.9)$ & $0.621^{*}$ \\
\hline \multicolumn{4}{|l|}{ Onset side } \\
\hline Right (n) & 10 & 7 & $0.345 \dagger$ \\
\hline Left (n) & 11 & 14 & \\
\hline UPDRS & $34.6(15.3)$ & $38.1(17.8)$ & $0.505^{*}$ \\
\hline $\mathrm{ADL}$ & $74.3(18.3)$ & $70.9(21.4)$ & $0.591^{*}$ \\
\hline Hoehn and Yahr score & $2.1(1.2)$ & $2.1(1.2)$ & $1.000^{*}$ \\
\hline 1 (n) & 8 & 9 & $0.927 \dagger$ \\
\hline $2(n)$ & 6 & 5 & \\
\hline 3 (n) & 3 & 2 & \\
\hline $4(n)$ & 4 & 5 & \\
\hline \multicolumn{4}{|l|}{ Drugs } \\
\hline Levodopa (n) & $18 / 21$ & $16 / 21$ & $0.697 \ddagger$ \\
\hline Daily dose (mg) & $472.2(145.8)$ & 546.9 (198.3) & $0.217^{*}$ \\
\hline \multicolumn{4}{|c|}{$\begin{array}{l}\text { Values are mean (SD) or } n \text { where specified. } \\
\text { *Student's } t \text { test for independent samples. } \\
\dagger \chi^{2} \text { test. } \\
\text { tFisher's exact test. } \\
\text { ADL, activities of daily living; BDI, Beck depression inventory; HDRS, Hamilton rating scale for depression; MMSE, } \\
\text { mini-mental state examination; UPDRS, unified Parkinson disease rating scale. }\end{array}$} \\
\hline
\end{tabular}



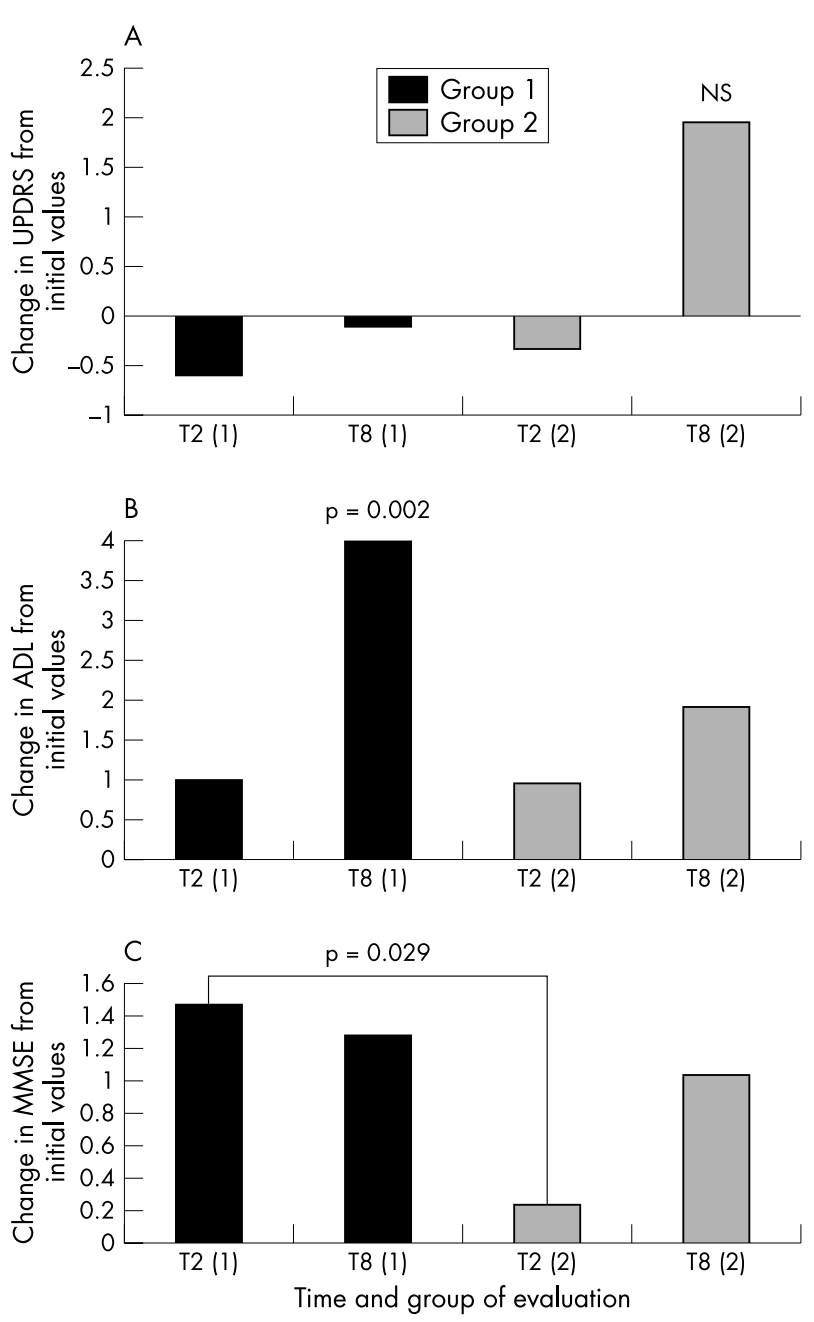

Figure 1 Changes in motor (UPDRS and ADL) and cognitive (MMSE) scores between week 2 (T2) and week 8 (T8) compared with baseline. (A) Changes in UPDRS from baseline, although not statistically significant, showed a trend of a worsening of motor function in group 2 after eight weeks ( $p>0.05 v$ baseline UPDRS). (B) Changes in ADL from baseline showing that group 1 had a significant improvement in ADL by week 8 ( $p=0.002 v$ baseline ADL). (C) Changes in MMSE from baseline showing a significant difference between group 1 and 2 after two weeks of treatment $(p=0.029)$; group 1 had significantly greater improvement than group 2 at week 2 . Tukey-HSD was used in the post hoc analysis. $A D L$, activities of daily living; MMSE, mini-mental state examination; UPDRS, unified Parkinson's disease rating scale.

For ADL, repeated measures of ANOVA showed a time effect $(F=9.170, p<0.001)$. On post hoc analysis, only group 1 had significantly higher scores of ADL at eight weeks compared with the initial value $(\mathrm{T} 0=74.0(18.8)$; $\mathrm{T} 8=78.0$ (17.0), $\mathrm{p}=0.002$ ) (fig lB).

Initial MMSE scores were 24.8 (4.5) and 24.1 (3.6) for groups 1 and 2, respectively. The low baseline MMSE scores could be explained by a low level of education in these patients, matching the MMSE average in this population. ${ }^{17}$ Repeated measures ANOVA for MMSE scores showed a significant effect of time ( $F=3.939, p=0.023$, ANOVA). On post hoc analysis, there was a significant difference between the two groups at week $2(\mathrm{p}=0.029)$, group $\mathrm{l}$ showing greater improvement in the MMSE scores than group 2. After eight weeks there was no difference between groups $(\mathrm{p}=0.674)$, suggesting that group 1 improved faster, but not overall more than group 2 (fig lC).
Adverse effects were more common in group 2 than in group $1 \quad\left(\chi^{2}=4.291, p=0.03,1 d f\right)$. The total numbers of complaints related to treatment were 40 and 53 after two weeks, and 14 and 40 after eight weeks in groups 1 and 2, respectively.

\section{DISCUSSION}

Our study shows that rTMS plus placebo has the same efficacy as fluoxetine plus sham rTMS for treating depression in patients with Parkinson's disease and is better tolerated. The drawbacks of each treatment have to be considered, as rTMS involves higher costs (TMS equipment, trained people to apply) and inconveniences (dislocation to treatment centre), and fluoxetine has some adverse effects, being associated with worsening of the motor symptoms of Parkinson's disease ${ }^{18}$ although this is still controversial. ${ }^{19}$

An important consideration regarding the study design relates to the choice of control groups. We chose an active control group instead of a placebo group, as it would be unethical to leave patients for such a long period without antidepressant treatment, although patients in the active rTMS arm could have been undertreated; our review ethics committee considered it inappropriate to add a third control group (sham rTMS plus placebo drug), and fluoxetine has been shown to be superior to placebo in a previous randomised trial, ${ }^{20}$ although a geriatric population was studied rather than patients with Parkinson's disease.

A methodological problem of rTMS studies is the sham coil. An ideal sham coil should produce the same scalp sensation (stimulation of the superficial nerves and muscles), a similar acoustic effect, no cortical effect, and have a similar coil shape compared with an active coil. ${ }^{21}$ The available sham coils do not produce the same scalp sensation, although this can be achieved by simultaneous peripheral electrical stimulation. ${ }^{22}$ In this way the patient could not fell the difference between active and sham coils. Given that all our patients were naive to rTMS, it is unlikely that they could have guessed the type of treatment.

Our study showed that a 10 day course of rTMS had an antidepressant effect that lasted for at least eight weeks. A long lasting antidepressant effect of a two week course of rTMS was previously demonstrated at three ${ }^{3}$ and $\operatorname{six}^{23}$ months of follow up. Although a 10 day course of rTMS has previously been found effective for treatment of depression, ${ }^{2-4}$ it was recently verified that a longer period of treatment confers additional benefit. ${ }^{24}$ We still know very little about how to choose suitable stimulation parameters (number of stimuli per session, number of sessions per week, optimum length of a course of stimulation). A longer period of rTMS treatment might have resulted in further improvement in depression in our patients and would be worth investigating in a similar population.

\section{Authors' affiliations}

F Fregni, A Pascual-Leone, Laboratory for Magnetic Brain Stimulation, Department of Neurology, Beth Israel Deaconess Medical Center and Harvard Medical School, Boston, Massachusetts, USA

C M Santos, M L Myczkowski, R Rigolino, J Gallucci-Neto, E R Barbosa, K D Valente, M A Marcolin, Psychiatry and Neurology Institute, Clinics Hospital, University of Sao Paulo, SP, Brazil

Competing interests: none declared

Correspondence to: Dr F Fregni, Department of Neurology, Beth Israel Deaconess Medical Center, 330 Brookline Ave \#KS 454, Boston, MA 02215, USA; ffregni@bidmc.harvard.edu

Received 28 August 2003

Revised 18 November 2003

Accepted 23 November 2003 


\section{REFERENCES}

1 Pascual-Leone A, Bartres-Faz D, Keenan J. Transcranial magnetic stimulation: studying the brain-behaviour relationship by induction of "virtual lesions". Philos Trans R Soc Lond B Biol Sci 1999;354:1229-38.

2 Pascual-Leone A, Rubio B, Pallardo F, et al. Rapid-rate transcranial magnetic stimulation of left dorsolateral prefrontal cortex in drug-resistant depression. Lancet 1996;348:233-7.

3 Triggs W, McCoy K, Greer R, et al. Effects of left frontal transcranial magnetic stimulation on depressed mood, cognition, and corticomotor threshold. Biol Psychiatry 1999;45:1440-6.

4 George M, Wassermann E, Williams W, et al. Changes in mood and hormone levels after rapid-rate transcranial magnetic stimulation (rTMS) of the prefrontal cortex. J Neuropsychiatry Clin Neurosci 1996;8:172-80.

5 Garcia-Toro M, Pascual-Leone A, Romera M, et al. Prefrontal repetitive transcranial magnetic stimulation as add on treatment in depression. I Neurol Neurosurg Psychiatry 2001;71:546-8.

6 Loo C, Mitchell P, Sachdev P, et al. Double-blind controlled investigation of transcranial magnetic stimulation for the treatment of resistant major depression. Am J Psychiatry 1999;156:946-8.

7 Gur E, Lerer B, Dremencov E, et al. Chronic repetitive transcranial magnetic stimulation induces subsensitivity of presynaptic serotonergic autoreceptor activity in rat brain. Neuroreport 2000;11:2925-9.

8 Ben-Shachar D, Belmaker R, Grisaru N, et al. Transcranial magnetic stimulation induces alterations in brain monoamines. J Neural Transm 1997; 104:191-7.

9 Ikeguchi M, Touge T, Nishiyama Y, et al. Effects of successive repetitive transcranial magnetic stimulation on motor performances and brain perfusion in idiopathic Parkinson's disease. J Neurol Sci 2003;209:41-6.

10 Shimamoto H, Takasaki K, Shigemori M, et al. Therapeutic effect and mechanism of repetitive transcranial magnetic stimulation in Parkinson's disease. J Neurol 2001;248:III48-52.

11 Dragasevic N, Potrebic A, Damianovic A, et al. Therapeutic efficacy of bilateral prefrontal slow repetitive transcranial magnetic stimulation in depressed patients with Parkinson's disease: an open study. Mov Disord 2002;17:528-32.

12 Starkstein S, Preziosi T, Forrester A, et al. Specificity of affective and autonomic symptoms of depression in Parkinson's disease. J Neurol Neurosurg Psychiatry 1990;53:869-73.
13 Lingiaerde $\mathbf{O}$, Ahlfors UG, Bech $\mathrm{P}$, et al. The UKU side effect rating scale. A new comprehensive rating scale for psychotropic drugs and a cross sectional study of side effects in neuroleptic treated patients. Acta Psychiatr Scand Suppl 1987:334:1-100

14 Sachdev P, McBride R, Loo $C$, et al. Effects of different frequencies of transcranial magnetic stimulation (TMS) on the forced swim test model of depression in rats. Biol Psychiatry 2002;51:474-9.

15 Wassermann E. Risk and safety of repetitive transcranial magnetic stimulation: report and suggested guidelines from the International Workshop on the Safety of Repetitive Transcranial Magnetic Stimulation, June 5-7, 1996. Electroencephalogr Clin Neurophysiol 1998;108:1-16.

16 McDonald W, Richard I, DeLong M. Prevalence, etiology, and treatment of depression in Parkinson's disease. Biol Psychiatry 2003;54:363-75.

17 Brucki S, Nitrini R, Caramelli P, et al. Suggestions for utilization of the mini-mental state examination in Brazil. Arq Neuropsiquiatr 2003;61:777-81.

18 Dell'Agnello G, Ceravolo R, Nuti A, et al. SSRIs do not worsen Parkinson's disease: evidence from an open-label, prospective study. Clin Neuropharmacol 2001;24:221-27.

19 Tollefson G, Bosomworth J, Heiligenstein J, et al. A double-blind, placebocontrolled clinical trial of fluoxetine in geriatric patients with major depression. Int Psychogeriatr 1995;7:89-104.

20 Tollefson G, Bosomworth J, Heiligenstein J, et al. A double-blind, placebo-controlled clinical trial of fluoxetine in geriatric patients with major depression. The Fluoxetine Collaborative Study Group. Int Psychogeriatr 1995; 7:89-104.

21 Loo C, Taylor J, Gandevia S, et al. Transcranial magnetic stimulation (TMS) in controlled treatment studies: are some "sham" forms active? Biol Psychiatry 2000:47:325-31.

22 Okabe $\mathrm{S}$, Ugawa $\mathrm{Y}$, Kanazawa I. $0.2-\mathrm{Hz}$ repetitive transcranial magnetic stimulation has no add-on effects as compared to a realistic sham stimulation in Parkinson's disease. Mov Disord 2003;18:382-8.

23 Dannon P, Dolberg O, Schreiber S, et al. Three and six-month outcome following courses of either ECT or rTMS in a population of severely depressed individuals - preliminary report. Biol Psychiatry 2002;51:687-90

24 Fitzgerald P, Brown T, Marston N, et al. Transcranial magnetic stimulation in the treatment of depression: a double-blind, placebo-controlled trial. Arch Gen Psychiatry 2003;60:1002-8. 